Kui Denga, Chunhua Hea , Jun Zhu, Juan Liang, Xiaohong Li, Xiaoyan Xie, Ping Yu, Nana Li, Qi Li* and Yanping Wang*

\title{
Incidence of congenital hypothyroidism in China: data from the national newborn screening program, 2013-2015
}

https://doi.org/10.1515/jpem-2017-0361

Received September 14, 2017; accepted April 3, 2018; previously published online May 1, 2018

\begin{abstract}
Background: Congenital hypothyroidism $(\mathrm{CH})$ is one of the most frequent, preventable causes of mental retardation. Little has been reported on the epidemiological characteristics of $\mathrm{CH}$ in China. We aimed to estimate the incidence of $\mathrm{CH}$ in China and investigate its geographical variation.
\end{abstract}

Methods: We analyzed data from the nationwide newborn screening program for CH between 2013 and 2015. Poisson regression was used to generate the odds ratios (ORs) and 95\% confidence intervals (CIs) between the rates of $\mathrm{CH}$ and selected demographic characteristics and assess the potential association between $\mathrm{CH}$ incidence and geographical locations.

Results: A total of 18,666 patients with $\mathrm{CH}$ were identified from 45.2 million newborns, yielding an overall incidence rate of 4.13 per 10,000 live births. Compared with those in the remote area, regardless of infant sex, a higher incidence risk for $\mathrm{CH}$ was present in newborns in coastal areas and inland areas (females: $\mathrm{OR}=2.00,95 \% \mathrm{CI}$ : 1.86 2.16 and $\mathrm{OR}=1.74,95 \% \mathrm{CI}: 1.61-1.87$, respectively; males: $\mathrm{OR}=1.70,95 \% \mathrm{CI}: 1.59-1.83$ and $\mathrm{OR}=1.52$, 95\% CI: 1.42 1.63 , respectively). Additionally, the highest risk of $\mathrm{CH}$

${ }^{\mathrm{a} K u i}$ Deng and Chunhua He contributed equally to this work. *Corresponding authors: Qi Li, Department of Pediatrics, West China Second University Hospital, Sichuan University, 20, Section 3, Ren Min South Road, Chengdu, Sichuan, P.R. China,

Phone: 86-028-85501363, Fax: 86-028-85501386,

E-mail: liqi210@126.com; and National Center for Birth Defects Monitoring, West China Second University Hospital, Sichuan University, Chengdu, Sichuan, P.R. China; and Yanping Wang, National Center for Birth Defects Monitoring of China, West China Second University Hospital, Sichuan University, 17, Section 3, Ren Min South Road, Chengdu, Sichuan, P.R. China, Phone: 86-028-85501189, Fax: 86-028-85501386, E-mail: wyxyanping@163.com; and Key Laboratory of Birth Defects and Related Diseases of Women and Children (Sichuan University), Ministry of Education, Chengdu, P.R. China for thyroid-stimulating hormone (TSH) screening values $<40 \mathrm{mU} / \mathrm{L}$ was observed among neonates in the coastal areas, while TSH screening values of 70-100 mU/L were observed among those in the inland areas.

Conclusions: The overall incidence of $\mathrm{CH}$ is high in China. The significant geographical variations of $\mathrm{CH}$ incidence are presented in this study.

Keywords: congenital hypothyroidism; geographical; incidence; newborn screening; thyroid-stimulating hormone.

\section{Introduction}

Congenital hypothyroidism $(\mathrm{CH})$ is a common endocrine disorder characterized as a thyroid hormone deficiency present at birth [1]. If treatment is delayed or left untreated, $\mathrm{CH}$ may result in impaired neurological development and intellectual disabilities [2-4]. Universal newborn screening programs through early identification have provided an opportunity for prompt thyroid hormone therapy for $\mathrm{CH}$ in the early days of life, thus preventing mental retardation and reducing the morbidity of related neurodevelopmental complications.

$\mathrm{CH}$ affects approximately one in 2000-4000 live births worldwide [5-10]. A significant variation in the ethnicity-specific incidence of $\mathrm{CH}$ has been reported in

Kui Deng: National Center for Birth Defects Monitoring, West China Second University Hospital, Sichuan University, Chengdu, Sichuan, P.R. China; Department of Pediatrics, West China Second University Hospital, Sichuan University, Chengdu, Sichuan, P.R. China; and Key Laboratory of Birth Defects and Related Diseases of Women and Children (Sichuan University), Ministry of Education, Chengdu, P.R. China. http://orcid.org/0000-0002-7716-6485 Chunhua He: National Center for Birth Defects Monitoring, West China Second University Hospital, Sichuan University, Chengdu, Sichuan, P.R. China; and Department of Pediatrics, West China Second University Hospital, Sichuan University, Chengdu, Sichuan, P.R. China Jun Zhu, Juan Liang, Xiaohong Li, Xiaoyan Xie, Ping Yu and Nana Li: National Center for Birth Defects Monitoring, West China Second University Hospital, Sichuan University, Chengdu, Sichuan, P.R. China 
previous studies $[5,9,11]$. There is a female preponderance for $\mathrm{CH}$, with a female-to-male ratio of 2:1 among thyroid dysgenesis but an approximate 1:1 ratio among eutopic thyroid patients [5, 12-15]. In addition, $\mathrm{CH}$ has been associated with adverse pregnancy outcomes, including birth defects, low birth weight and preterm birth $[5,11,16,17]$. Moreover, $\mathrm{CH}$ may result from the changes of some environmental factors, including iodine deficiency/excess, and seasonal patterns [18-23]. Recently, a number of epidemiological studies have indicated that the incidence of $\mathrm{CH}$ is increasing in multiple regions over the past two decades [5-7, 24-26]. The most predominant causes of an increase in $\mathrm{CH}$ may be a reduction of the thyroid-stimulating hormone (TSH) cutoff (CO) levels and a growing number of preterm births. An increased proportion of $\mathrm{CH}$ with eutopic glands may also account for this trend. However, other related explanations on this trend, e.g. increasing exposure to environmental risk factors, show inconsistencies.

Although multiple studies have demonstrated that $\mathrm{CH}$ is more common among Asian populations than in some Western newborn populations, epidemiological studies regarding the incidence of $\mathrm{CH}$ in Asian countries, particularly in China, are relatively limited. The newborn screening program for $\mathrm{CH}$ was initiated as a pilot study in the 1980s in two eastern provinces (Beijing, Shanghai) in China. Until 2015, the newborn screening program was implemented throughout the country and performed in 95\% of districts/counties of 31 provinces (Hong Kong, Taiwan and Macao not included). A total of 230 newborn screening centers were developed, covering approximately $90 \%$ of the newborn population in China (covering nearly $100 \%$ in the eastern regions).

Due to scarce nationwide data available on $\mathrm{CH}$ incidence, the aim of the present study was to estimate the incidence of $\mathrm{CH}$ using the largest dataset from the national newborn CH screening information system during 2013 to 2015 in China. We also described the geographic variation in the incidence of $\mathrm{CH}$.

\section{Materials and methods}

\section{Chinese $\mathrm{CH}$ newborn screening program}

The national newborn screening program in China is centrally administered by the National Health and Family Planning Commission, which is responsible for drafting related neonatal screening policies, plans, and technical standards and norms. In each province, local newborn screening centers (LNBSCs) in charge of sample testing, diagnosis, treatment and follow-up are authorized and administered by provincial health departments (Figure 1). Subsequently, a provincial newborn screening center (PNBSC) was established to guide and supervise the LNBSCs for all neonatal screening practices. In 2003, the Ministry of Health issued the Technological Guideline on National Newborn Screening (TGNNBS) to advance the administrative management and standardization on neonatal screening.

According to the TGNNBS, whole-blood TSH was measured as a primary marker for neonatal $\mathrm{CH}$ screening. Whole blood, heel-prick samples were collected by well-trained staff in maternity hospitals on filter papers from newborns between $72 \mathrm{~h}$ and 7 days after birth. Premature, low birth weight or sick neonates and those who were discharged from the maternity hospitals before $72 \mathrm{~h}$ of life were sampled within 20 days after delivery for $\mathrm{CH}$ screening. The whole blood samples were dried at room temperature and then sent to the LNBSCs for testing within 5 workdays. All screening laboratories were required to process and analyze the samples within 5 workdays after receiving samples and immediately report the positive cases. TSH was qualified through dissociation-enhanced lanthanide fluoroimmunoassay (DELFIA) in most of the laboratories, while enzyme-linked immunosorbent assay (ELISA) and enzymatic immunofluorescence assay (EFIA) were used in a minority of laboratories, with CO levels varying from 10 to $20 \mathrm{mU} / \mathrm{L}$ whole blood. Each laboratory set the TSH CO levels based on their respective TSH percentiles. Any cases showing higher TSH CO levels through double testing were followed-up and subjected to further diagnosis. In each LNBSC, the diagnosis of $\mathrm{CH}$ was confirmed by trained pediatric endocrinologists according to the thyroid function on serum (serum TSH elevated and serum-free thyroxine reduced). Further examinations, including thyroid ultrasound and/or scintigraphy, were generally performed to complete the diagnosis of $\mathrm{CH}$. The pediatric endocrinologists were required to follow the above guidelines for the diagnosis of $\mathrm{CH}$. A written informed consent on $\mathrm{CH}$ screening was obtained from parents prior to the collection of blood samples from the neonates.

\section{Data collection}

The data in the present analysis were derived from the Chinese Newborn Screening Information System (CNBSIS) between 2013 and 2015. This system was performed by the National Office of Maternal and Child Health Surveillance (NOMCHS) for routinely collecting and reporting basic information on the testing, diagnosis and follow-up of infants with $\mathrm{CH}$ and was utilized by all screening centers, including PNBSCs and LNBSCs. In every LNBSC, trained staff were assigned to complete the online forms quarterly based on the blood sample cards, laboratory test reports and medical records, including data on the number of neonates screened (total, geographical-specific and sex-specific), the number of neonates diagnosed as $\mathrm{CH}$ and the demographic and clinical characteristics of $\mathrm{CH}$ infants (geographical location, date of birth, sex, TSH screening values and cases diagnosis). All forms were reviewed at the provincial level by professionals in the PNBSCs responsible for data quality. Incomplete forms and nonspecific records were returned to the LNBSCs and verified quarterly. Additionally, a provincial annual form was collected yearly by trained staff in the PNBSCs. Then, the data were reported to the NOMCHS (national level), where an expert group, composed of pediatric endocrinologists, biostatisticians and epidemiologists, was responsible for diagnosis confirmation, data reviewing and compilation. In the present study, data from Tibet was not included because 


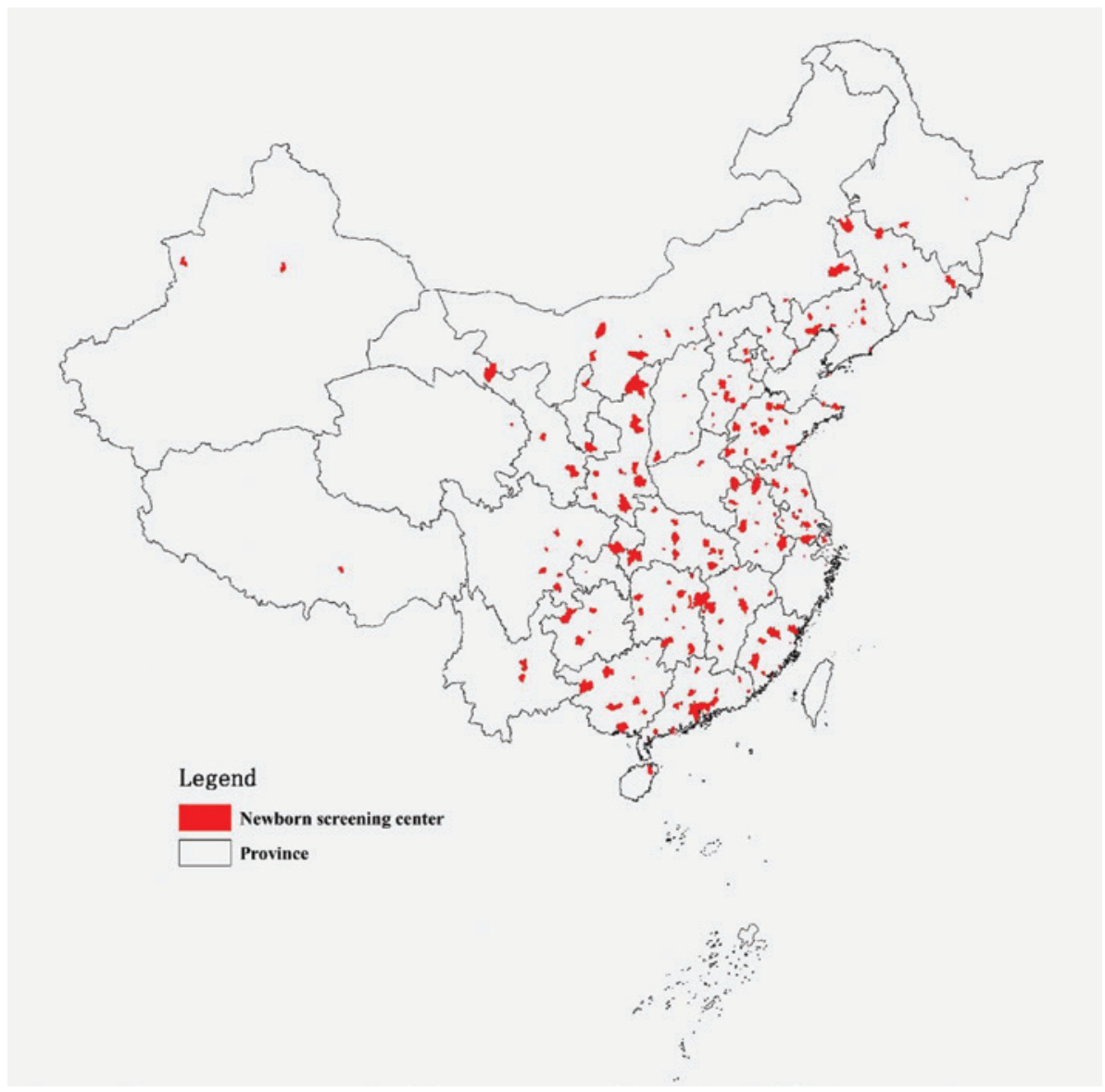

Figure 1: Distribution of newborn screening centers in China, 2015.

The number of newborn screening centers in the coastal, inland and remote areas were 84, 104 and 42, respectively. Red: the county in which newborn screening center is located.

CH screening there was initiated in 2015. The study was approved by the Ethics Committee of West China Second University Hospital, Sichuan University, China, and performed in accordance with the Declaration of Helsinki, 1964 and its subsequent amendments or comparable ethical standards.

\section{Quality control}

Since 1998, the LNBSCs received annual laboratory quality control for neonatal $\mathrm{CH}$ screening by the National Center for Clinical Laboratory (NCCL). The NCCL was authorized to convene an annual conference on the quality evaluation and monitoring of practices among laboratories to ensure their quality on $\mathrm{CH}$ screening. Some laboratories also participated in external quality assessment under the supervision of Centers for Disease Control and Prevention (CDC) in the United States [27]. Additionally, field supervision at the LNBSCs under the responsibility of the NMCHSO was performed annually by a national expert group composed of pediatric endocrinologists, laboratory technicians and biostatisticians to evaluate the practice of $\mathrm{CH}$ screening and the completeness, accuracy and timeliness of the data in the CNBSIS via medical records reviews and face-to-face interviews with related staff.

\section{Statistical analysis}

In the present analysis, 30 provinces were categorized according to the geographical locations and socioeconomic status into the coastal area (Beijing, Tianjin, Shanghai, Liaoning, Shandong, Jiangsu, Zhejiang, Fujian and Guangdong), inland area (Heilongjiang, Jilin, Hebei, Henan, Shanxi, Anhui, Hubei, Hunan, Guangxi, Shaanxi, Jiangxi, Hainan and Chongqing) and remote area (Inner Mongolia, Ningxia, Gansu, Xinjiang, Qinghai, Yunnan, Guizhou and Sichuan). The incidence of overall $\mathrm{CH}$ by geographical locations (coastal area, inland area and remote area), infant sex (female and male) and the year of infant birth (2013, 2014 and 2015), expressed as the number per 10,000 live births, was calculated as the number of diagnosed cases divided by the number of screened newborns. The $95 \%$ confidence intervals (CIs) of $\mathrm{CH}$ incidence were estimated based on a Poisson distribution. Poisson regression was used to generate the odds ratios (ORs) and 95\% CIs between the rates of $\mathrm{CH}$ and selected demographic characteristics and assess the potential association between $\mathrm{CH}$ incidence and geographical locations while controlling for other demographic factors. To further analyze the geographical variation of $\mathrm{CH}$, cases were grouped by the TSH screening values at an interval of $10 \mathrm{mU} / \mathrm{L}$. Poisson regression was also used to assess the geographical difference of 
$\mathrm{CH}$ based on the TSH screening values. All tests of hypotheses were two-tailed with a type 1 error rate fixed at $5 \%$. Statistical analyses were performed using SAS 9.1 software (SAS Institute Inc., Cary, NC, USA).

\section{Results}

From 2013 to 2015, approximately 45.2 million newborns were screened in China. The percentage of screened newborns was highest in the coastal area (90\%), followed by the inland area (88\%). A total of 18,666 cases were identified as $\mathrm{CH}$, yielding an overall incidence rate of 4.13 per 10,000 screened newborns. Among the identified $\mathrm{CH}$ cases, 9364 cases occurred in males (overall incidence 3.87 per 10,000), whereas 9294 cases were in females (overall incidence 4.44 per 10,000). The female-to-male ratio for $\mathrm{CH}$ cases was 0.99 . However, females were more likely to have $\mathrm{CH}$ than males $(\mathrm{OR}=1.15$; 95\% CI: $1.12-1.18)$ (Table 1).
Table 1 also presents the geographical and annual incidence of CH between 2013 and 2015. During the 3-year study period, the $\mathrm{CH}$ incidence predominated in the coastal area, followed by that in the inland area. Compared with that in the remote area, a higher incidence risk of $\mathrm{CH}$ was observed in the coastal area and the inland area $(\mathrm{OR}=1.84$, 95\% CI: $1.75-1.93$ and $\mathrm{OR}=1.62$, 95\% CI: $1.54-$ 1.70, respectively). According to the years of birth, the incidence rates of $\mathrm{CH}$ were 4.05, 4.15 and 4.20 per 10,000 live births in 2013, 2014 and 2015, respectively. There was no statistically significant difference for $\mathrm{CH}$ incidence among different groups by years of birth.

Table 2 shows the incidence of $\mathrm{CH}$ according to infant sex and geographical locations between 2013 and 2015. Females located in coastal areas had the highest $\mathrm{CH}$ incidence, at 5.02 per 10,000 newborns screened, followed by males in coastal areas $(4.49$ per 10,000$)$ and females in inland areas (4.49 per 10,000). Compared with that in remote areas, regardless of infant sex, a higher incidence of $\mathrm{CH}$ was present in newborns in coastal areas and in

Table 1: Incidence rates of CH by sex, geographical locations and years of birth in China, 2013-2015.

\begin{tabular}{|c|c|c|c|c|}
\hline & $n(\%)^{a}$ & $\mathrm{CH}^{\mathrm{b}}$ & $\begin{array}{r}\text { Incidence per } 10,000 \text { screened } \\
\text { newborns }(95 \% \mathrm{Cl})\end{array}$ & OR $(95 \% \mathrm{Cl})$ \\
\hline Total & $45,151,947(90)$ & 18,666 & $4.13(4.08-4.19)$ & \\
\hline \multicolumn{5}{|l|}{ Sex } \\
\hline Male & $24,183,956^{d}$ & 9364 & $3.87(3.79-3.95)$ & Ref. \\
\hline Female & $20,913,113^{d}$ & 9294 & $4.44(4.35-4.54)$ & $1.15(1.12-1.18)$ \\
\hline \multicolumn{5}{|c|}{ Geographical locations } \\
\hline Remote area & $7,042,884(80)$ & 1814 & $2.58(2.46-2.70)$ & Ref. \\
\hline Inland area & $20,760,725(88)$ & 8646 & $4.16(4.08-4.25)$ & $1.62(1.54-1.70)$ \\
\hline Coastal area & $17,348,338(99)$ & 8206 & $4.73(4.63-4.83)$ & $1.84(1.75-1.93)$ \\
\hline \multicolumn{5}{|l|}{ Years of birth } \\
\hline 2013 & $14,705,320(90)$ & 5959 & $4.05(3.95-4.16)$ & Ref. \\
\hline 2014 & $15,356,369(89)$ & 6368 & $4.15(4.05-4.25)$ & $1.02(0.99-1.06)$ \\
\hline 2015 & $15,090,258(92)$ & 6339 & $4.20(4.10-4.31)$ & $1.04(1.00-1.07)$ \\
\hline
\end{tabular}

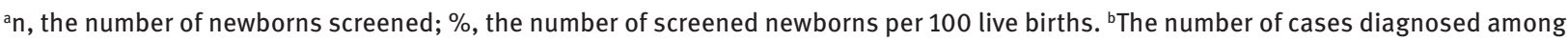
screened newborns. 'A total of 54,878 newborns (eight cases included) were excluded from our analysis because information on sex were missed or unknown. ${ }^{\mathrm{d} T h e}$ percentages of screened newborns by sex were unknown because the number of sex-specific live births were missed in the present system. $\mathrm{CH}$, congenital hypothyroidism; OR, odds ratio; $\mathrm{Cl}$, confidence interval; Ref., reference group.

Table 2: Incidence rates of CH by infant sex and geographical locations, in China, 2013-2015.

\begin{tabular}{|c|c|c|c|c|c|c|}
\hline \multirow[t]{2}{*}{ Geographical locations } & \multicolumn{3}{|l|}{ Male } & \multicolumn{3}{|l|}{ Female } \\
\hline & Cases & Rates $^{a}$ & OR $(95 \% \mathrm{Cl})$ & Cases & Rates $^{\mathrm{a}}$ & OR $(95 \% \mathrm{Cl})$ \\
\hline Remote area & 831 & 2.24 & Ref & 983 & 2.95 & Ref \\
\hline Inland area & 4316 & 3.89 & $1.74(1.61-1.87)$ & 4324 & 4.49 & $1.52(1.42-1.63)$ \\
\hline Coastal area & 4217 & 4.49 & $2.00(1.86-2.16)$ & 3987 & 5.02 & $1.70(1.59-1.83)$ \\
\hline
\end{tabular}

a Rate is the number of cases per 10,000 screened newborns. OR, odds ratio; $\mathrm{Cl}$, confidence interval; Ref., reference group. 
inland areas (females: $\mathrm{OR}=2.00,95 \% \mathrm{CI}$ : $1.86-2.16$ and $\mathrm{OR}=1.74,95 \% \mathrm{CI}: 1.61-1.87$, respectively; males: $\mathrm{OR}=1.70$, 95\% CI: $1.59-1.83$ and OR $=1.52$, 95\% CI: $1.42-1.63$, respectively). Additionally, regardless of the infant sex, infants located in coastal areas were more likely to have $\mathrm{CH}$ than those in inland areas $(\mathrm{p}<0.0001)$.

The incidence rates of $\mathrm{CH}$ according to the TSH screening values and geographical locations in China over the study period are shown in Figure 2. A U-shaped distribution was observed between $\mathrm{CH}$ incidence and TSH screening values in different regions. Higher incidence rates of $\mathrm{CH}$ were observed among newborns with TSH screening values $<30 \mathrm{mU} / \mathrm{L}$ and $>100 \mathrm{mU} / \mathrm{L}$. For any TSH screening value group, the incidence of $\mathrm{CH}$ in the remote areas was lower than that in the inland and coastal areas.

Figure 3 presents the ORs of $\mathrm{CH}$ by TSH screening values and geographical locations after adjusting for infant sex and years of birth. Compared with infants located in remote areas, those located in inland areas were more likely to have $\mathrm{CH}$ for any TSH screening value groups. The same difference was observed among infants located in the coastal area, except for TSH screening values of $70-80 \mathrm{mU} / \mathrm{L}$. For groups with TSH screening values $<40 \mathrm{mU} / \mathrm{L}$, infants located in the coastal area had the highest risk of $\mathrm{CH}$, followed by those in the inland area. However, the highest risk of $\mathrm{CH}$ was detected in the inland area for groups of TSH screening values of 70-100 mU/L. Additionally, for groups of TSH screening values $40-70 \mathrm{mU} / \mathrm{L}$, an elevated risk of $\mathrm{CH}$ was observed in the coastal area compared with that in the inland area, but this difference was not statistically significant ( $p>0.05)$ (data not shown).

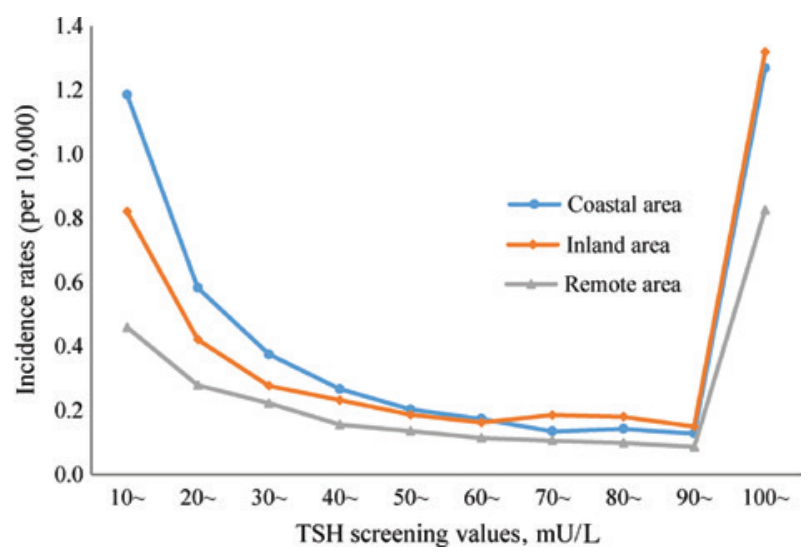

Figure 2: Incidence rates of $\mathrm{CH}$ by TSH screening values and geographical locations, in China, 2013-2015.

TSH, thyroid-stimulating hormone.

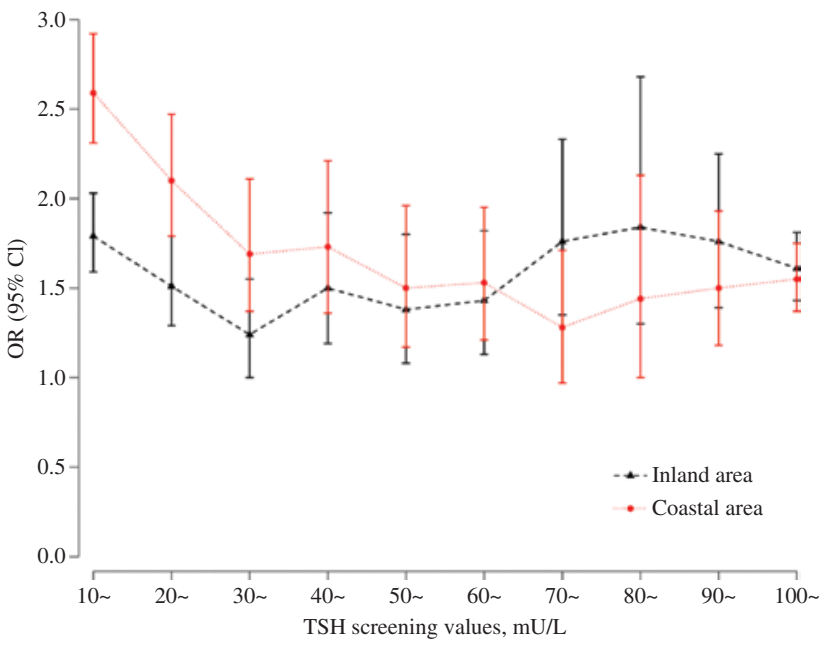

Figure 3: $\mathrm{ORs}$ of $\mathrm{CH}$ by TSH screening value and geographical location, in China, 2013-2015.

ORs were adjusted by infant sex and years of birth. The incidence of $\mathrm{CH}$ in the remote area was the reference group. TSH, thyroid-stimulating hormone; $\mathrm{Cl}$, confidence interval.

\section{Discussion}

The present study used the largest national database on newborn screening to investigate an overall incidence of $\mathrm{CH}$ of 4.1 per 10,000 live births, and we also observed the geographic variations of $\mathrm{CH}$ incidence in China. The CNBSIS, the only nationwide network for routine data collection and reporting on newborn screening, provided a large sample size and a sound representative population to assess the estimates of the incidence of $\mathrm{CH}$.

Previous studies have demonstrated that the incidence of $\mathrm{CH}$ varies according to race and ethnicity, with a higher incidence in Asians than in non-Hispanic Whites and non-Hispanic Blacks. A recent report in California during 2001-2007 revealed that $\mathrm{CH}$ incidence is more common in Asian Indians (5.7 per 10,000 newborns) and in Chinese and Vietnamese (4.2 per 10,000 newborns) than in non-Hispanic Whites (3.6 per 10,000 newborns) and non-Hispanic Blacks (0.9 per 10,000 newborns) [9]. Similar results were also reported in previous studies [5, $11,28]$. In the present study, the overall incidence of $\mathrm{CH}$ in China was similar to the estimates of recent studies conducted in California [9] and New Zealand [29], confirming a higher incidence among Asians. However, similar or even higher results were found in Quebec (4.7 per 10,000 live births in 2009) [15], Italy (5.2 per 10,000 live births between 1999 and 2008) [16] and Greece (6.4 per 10,000 live births between 2000 and 2002) [30]. 
Few studies have presented the incidence of $\mathrm{CH}$ in China during the past two decades, although $\mathrm{CH}$ has been widely studied in Western countries. Two previous studies using data from the national network of neonatal screening centers collected by the National Center for Clinical Laboratory in China assessed the $\mathrm{CH}$ incidence of Chinese newborns, yielding the rates of 4.89 per 10,000 newborns from 1985 to 2007 and 4.88 per 10,000 newborns from 2000 to 2007 [27, 31]. However, due to a lower coverage for newborns screened in both studies, a limited representative population may underestimate or overestimate the true incidence rate of $\mathrm{CH}$. Both rates in these studies were higher than our overall rate but similar to the estimate of 4.73 per 10,000 in the coastal area reported in the present study. This discrepancy may be primarily explained by the higher population of newborns located in the eastern region and the lower population of newborns in the midwestern regions in these two studies than in the present study. In other Asian regions, much higher $\mathrm{CH}$ incidences were also observed in Taiwan [8] and Japan [32] (5.02 per 10,000 births during 1997-2008 and 6.8 per 10,000 live births during 1994-2002, respectively). These inconsistencies may be caused by the differences among the study populations, neonatal screening program practices and potential environmental risk factors.

Notably, due to unbalanced development, there are significant differences among regions (coastal area, inland area and remote area) in China regarding the progress on economic, culture, education and environment. In general, the coastal area is more developed than the inland and remote areas. The present study also observed a statistically significant geographic variation of $\mathrm{CH}$ incidences across regions, showing a higher incidence risk in the coastal area and in the inland area but a lower incidence risk in the remote area. This difference may be the result of several factors. First, some differences among regions, including screening program practices (e.g. TSH CO levels, screening methodology), follow-up of screening positive cases and laboratory testing, may be correlated with case identification and diagnosis for $\mathrm{CH}$. Generally, more improved screening activities are present in the coastal and inland areas than in remote areas. Therefore, cases have a higher chance to be identified and diagnosed from newborns in the coastal and inland areas. Second, the survival rates for preterm births across regions may lead to geographical variations in $\mathrm{CH}$ incidence, although there were no data available to support the differences between $\mathrm{CH}$ and preterm births by regions in China. Several previous studies conducted in Western countries have indicated that an elevated survival rate for preterm neonates contributes to an increasing $\mathrm{CH}$ incidence over years $[9,16]$. Because of more advanced neonatal health care in the coastal area and less advanced care in the remote area, a higher survival rate among preterm births may occur in the coastal area than in the remote area, suggesting a higher $\mathrm{CH}$ incidence in the coastal area. However, no data were available to identify whether prematurity contributed to the increased rate of $\mathrm{CH}$ in the coastal area. Third, several environmental risk factors may be associated with the incidence of $\mathrm{CH}$, such as iodine deficiency or iodine excess [33,34]. Although there is no evidence to support the geographical differences in the iodine nutritional status across regions in China, several studies have revealed that an iodine deficiency during pregnancy exists in the coastal areas of China [35, 36]. Additionally, differences in $\mathrm{CH}$ incidence in geographic regions may also be related to the distribution of population with $\mathrm{CH}$ susceptibility genes.

Furthermore, the present study showed a change in the geographical variation of the incidence according to the TSH screening value. In the present analysis, the geographical variation of the incidence was present among newborns with lower TSH screening values but also among those with higher TSH screening values. Therefore, the geographical variation of $\mathrm{CH}$ incidence may be correlated not only with the TSH CO levels and screening assay methods but also with other risk factors, such as preterm births, iodine deficiency/excess and other unknown environmental factors. Future epidemiologic studies are needed to emphasize that it is of great importance to analyze the distributions and identify potential risk factors of $\mathrm{CH}$ across regions.

There are some limitations in the present study. First, due to the limited screening ability in some centers, particularly in the remote areas, some mild cases were not detected via neonatal $\mathrm{CH}$ screening and some positive cases were not recalled for further diagnosis. Consequently, the rate reported in the present study may underestimate the true incidence of $\mathrm{CH}$ in China. Second, because the data on thyroid ultrasound and scintigraphy were not acquired in these systems, we could not analyze the demographic characteristics of thyroid dysgenesis and dyshormonogenesis. In the future, national newborn screening information system is needed to improve data access regarding the $\mathrm{CH}$ subtypes. Third, we did not collect more detailed information on neonatal $\mathrm{CH}$ screening procedures. Therefore, we could not obtain TSH CO levels and TSH assay methods across centers and could not describe the age at screening and the age at treatment initiation for $\mathrm{CH}$. We could also not, through the long-term follow-up data, identify cases of permanent and transient 
$\mathrm{CH}$. Thus, a detailed analysis of the screening procedures was not performed in the present study.

In conclusion, it is of great importance that newborn screening be implemented in China, as long-term neurodevelopmental consequences occur when $\mathrm{CH}$ is not treated early. There is room for improvement in the newborn screening program in China because not all areas have fully implemented this program. Furthermore, as the incidence of $\mathrm{CH}$ seems to be increased in the Chinese population, further studies are needed to identify the causes of the increased incidence of $\mathrm{CH}$ in China.

Acknowledgments: The authors would like to thank the nurses of the obstetric and pediatric departments from the maternity hospitals for the ongoing blood sample collection. The authors would also like to thank the laboratory technicians and pediatric endocrinologists from the newborn screening centers for case testing, diagnosis, treatment and follow-up and the staff of the newborn screening centers for continued collaboration and support for the national newborn screening database.

Author contributions: KD, JZ, JL and YPW contributed to the study design and manuscript drafting and revision. CHH, QL, XHL, XYX, PY and NNL assisted with the data review and coding. $\mathrm{KD}, \mathrm{CHH}, \mathrm{QL}$ and YPW contributed to the data analysis and interpretation. All authors reviewed and approved the final version of the manuscript. All the authors have accepted responsibility for the entire content of this submitted manuscript and approved submission.

Research funding: This study was supported in part through the National "Twelfth Five-Year" Plan for Science \& Technology Support (Grant ID: 2014BAI06B01) and the National "Thirteenth Five-Year” Key Technologies R\&D Program (Grant ID: 2017YFC1001704).

Employment or leadership: None declared.

Honorarium: None declared.

Competing interests: The funding organization(s) played no role in the study design; in the collection, analysis, and interpretation of data; in the writing of the report; or in the decision to submit the report for publication.

\section{References}

1. Gruters A, Krude H. Detection and treatment of congenital hypothyroidism. Nat Rev Endocrinol 2011;8:104-13.

2. Klein AH, Meltzer S, Kenny FM. Improved prognosis in congenital hypothyroidism treated before age three months. J Pediatr 1972;81:912-5.

3. Grosse SD, Van Vliet G. Prevention of intellectual disability through screening for congenital hypothyroidism: how much and at what level? Arch Dis Child 2011;96:374-9.
4. Rovet JF, Ehrlich R. Psychoeducational outcome in children with early-treated congenital hypothyroidism. Pediatrics 2000; 105(3 Pt 1):515-22.

5. Harris KB, Pass KA. Increase in congenital hypothyroidism in New York State and in the United States. Mol Genet Metab 2007;91:268-77.

6. Barry Y, Bonaldi C, Goulet V, Coutant R, Leger J, et al. Increased incidence of congenital hypothyroidism in France from 1982 to 2012: a nationwide multicenter analysis. Ann Epidemiol 2016;26:100-5; e101-4.

7. Mitrovic K, Vukovic R, Milenkovic T, Todorovic S, Radivojcevic J, et al. Changes in the incidence and etiology of congenital hypothyroidism detected during 30 years of a screening program in central Serbia. Eur J Pediatr 2016;175:253-9.

8. Chen CY, Lee KT, Lee CT, Lai WT, Huang YB. Epidemiology and clinical characteristics of congenital hypothyroidism in an Asian population: a nationwide population-based study. J Epidemiol 2013;23:85-94.

9. Hinton CF, Harris KB, Borgfeld L, Drummond-Borg M, Eaton R, et al. Trends in incidence rates of congenital hypothyroidism related to select demographic factors: data from the United States, California, Massachusetts, New York, and Texas. Pediatrics 2010;125(Suppl 2):S37-47.

10. Anastasovska V, Kocova M. Ethnicity and incidence of congenital hypothyroidism in the capital of Macedonia. J Pediatr Endocrinol Metab 2017;30:405-9.

11. Waller DK, Anderson JL, Lorey F, Cunningham GC. Risk factors for congenital hypothyroidism: an investigation of infant's birth weight, ethnicity, and gender in California, 1990-1998. Teratology 2000;62:36-41.

12. Padilla CD, Therrell BL. Newborn screening in the Asia Pacific region. J Inherit Metab Dis 2007;30:490-506.

13. Eugene D, Djemli A, Van Vliet G. Sexual dimorphism of thyroid function in newborns with congenital hypothyroidism. J Clin Endocrinol Metab 2005;90:2696-700.

14. Skordis N, Toumba M, Savva SC, Erakleous E, Topouzi M, et al. High prevalence of congenital hypothyroidism in the Greek Cypriot population: results of the neonatal screening program 1990-2000. J Pediatr Endocrinol Metab 2005;18:453-61.

15. Deladoey J, Ruel J, Giguere Y, Van Vliet G. Is the incidence of congenital hypothyroidism really increasing? A 20-year retrospective population-based study in Quebec. J Clin Endocrinol Metab 2011;96:2422-9.

16. Olivieri A, Fazzini C, Medda E; Italian Study Group for Congenital Hypothyroidism. Multiple factors influencing the incidence of congenital hypothyroidism detected by neonatal screening. Horm Res Paediatr 2015;83:86-93.

17. Medda E, Olivieri A, Stazi MA, Grandolfo ME, Fazzini C, et al. Risk factors for congenital hypothyroidism: results of a population casecontrol study (1997-2003). Eur J Endocrinol 2005;153:765-73.

18. Rezaeian S, Poorolajal J, Moghimbegi A, Esmailnasab N. Risk factors of congenital hypothyroidism using propensity score: a matched case-control study. J Res Health Sci 2013;13:151-6.

19. Gu YH, Kato T, Harada S, Inomata H, Saito T, et al. Seasonality in the incidence of congenital hypothyroidism in Japan: genderspecific patterns and correlation with temperature. Thyroid 2007;17:869-74.

20. Nakamizo M, Toyabe S, Asami T, Akazawa K. Seasonality in the incidence of congenital hypothyroidism in Japan. J Paediatr Child Health 2005;41:390-1. 
21. Coakley JC, Francis I, Gold H, Mathur K, Connelly JF. Transient primary hypothyroidism in the newborn: experience of the Victorian Neonatal Thyroid Screening Programme. Aust Paediatr J 1989;25:25-30.

22. Saglam H, Buyukuysal L, Koksal N, Ercan I, Tarim O. Increased incidence of congenital hypothyroidism due to iodine deficiency. Pediatr Int 2007;49:76-9.

23. Khanjani N, Ahmadzadeh A, Bakhtiari B, Madadizadeh F. The role of season and climate in the incidence of congenital hypothyroidism in Kerman province, Southeastern Iran. J Pediatr Endocrinol Metab 2017;30:149-57.

24. Kurinczuk JJ, Bower C, Lewis B, Byrne G. Congenital hypothyroidism in Western Australia 1981-1998. J Paediatr Child Health 2002;38:187-91.

25. Pearce MS, Korada M, Day J, Turner S, Allison D, et al. Increasing incidence, but lack of seasonality, of elevated TSH levels, on newborn screening, in the North of England. J Thyroid Res 2010;2010:101948.

26. Corbetta C, Weber G, Cortinovis F, Calebiro D, Passoni A, et al. A 7-year experience with low blood TSH cutoff levels for neonatal screening reveals an unsuspected frequency of congenital hypothyroidism (CH). Clin Endocrinol 2009;71:739-45.

27. Zhan JY, Qin YF, Zhao ZY. Neonatal screening for congenital hypothyroidism and phenylketonuria in China. World J Pediatr 2009;5:136-9.

28. Lorey FW, Cunningham GC. Birth prevalence of primary congenital hypothyroidism by sex and ethnicity. Hum Biol 1992;64:531-8.
29. Albert BB, Cutfield WS, Webster D, Carll J, Derraik JG, et al. Etiology of increasing incidence of congenital hypothyroidism in New Zealand from 1993-2010. J Clin Endocrinol Metab 2012;97:3155-60.

30. Mengreli C, Kanaka-Gantenbein C, Girginoudis P, Magiakou MA, Christakopoulou I, et al. Screening for congenital hypothyroidism: the significance of threshold limit in false-negative results. J Clin Endocrinol Metab 2010;95:4283-90.

31. Gu X, Wang Z, Ye J, Han L, Qiu W. Newborn screening in China: phenylketonuria, congenital hypothyroidism and expanded screening. Ann Acad Med Singapore 2008;37(12 Suppl):107-4.

32. Gu YH, Kato T, Harada S, Inomata H, Aoki K. Time trend and geographic distribution of treated patients with congenital hypothyroidism relative to the number of available endocrinologists in Japan. J Pediatr 2010;157:153-7.

33. American Academy of Pediatrics, Rose SR, Section on Endocrinology and Committee on Genetics, American Thyroid Association, Brown RS, et al. Update of newborn screening and therapy for congenital hypothyroidism. Pediatrics 2006;117:2290-303.

34. Du Y, Gao Y, Meng F, Liu S, Fan Z, et al. Iodine deficiency and excess coexist in China and induce thyroid dysfunction and disease: a cross-sectional study. PLoS One 2014;9:e111937.

35. Wei Z, Wang W, Zhang J, Zhang X, Jin L, et al. Urinary iodine level and its determinants in pregnant women of Shanghai, China. $\mathrm{Br}$ J Nutr 2015;113:1427-32.

36. Mao G, Ding G, Lou X, Zhang R, Zheng P, et al. Survey of iodine nutritional status in 2011, Zhejiang, China. Asia Pac J Clin Nutr 2015;24:234-44. 